\title{
Quadrupling the Data Rate for Narrowband Internet of Things Without Modulation Upgrade
}

\author{
Xinyue Liu and Izzat Darwazeh \\ Department of Electronic and Electrical Engineering \\ University College London \\ London WC1E 7JE, UK \\ Email: x.liu.17@ucl.ac.uk, i.darwazeh@ucl.ac.uk
}

\begin{abstract}
This work proposes a novel signalling method for Narrowband Internet of Things (NB-IoT) mobile system where a quadrupled data rate can be achieved by combining two orthogonal techniques; the frequency orthogonal Fast-Orthogonal Frequency Division Multiplexing (Fast-OFDM) scheme coupled with the time orthogonal Hilbert transform (HT) pair. In this paper, the orthogonality features of one dimension in Fast-OFDM and of the HT pair are explored. The newly designed system structure is presented where the HT pulse pair is generated by square root raised cosine (SRRC) filter with a matched configuration at the receiver. System performance is investigated in terms of bit error rate (BER), effective data rate and spectral efficiency. Simulation results show that the HT-Fast-OFDM system provides 4 times the data rate relative to an OFDM system employing the same modulation scheme and occupying the same bandwidth. Furthermore, the proposed system has compelling advantages over 16-QAM OFDM for it achieves a better BER performance at the same spectral efficiency.
\end{abstract}

Index Terms-Fast-OFDM, Hilbert transform pair, data rate, spectral efficiency, NB-IoT

\section{INTRODUCTION}

The International Telecommunication Union (ITU) has presented IMT-2020 to clarify the requirements for 5 th generation (5G) technologies. The Internet of Things (IoT), is regarded as the major paradigm shift from the 4th Generation (4G) to the undergoing 5G system [1]. Although current IoT research focuses on improving the power efficiency of the legacy wireless systems, the trade-off between the demand for highspeed transmission and the scarcity of wireless spectrum still exists in this area. With such trade-off, 5G networks may require new waveform designs and transceiver structures to improve the spectral efficiency.

Orthogonal Frequency Division Multiplexing (OFDM) is the dominant signalling format adopted in LTE standard for 4th generation $(4 \mathrm{G})$ cellular mobile network [2]. It also forms the basis of Wi-Fi [3], WiMAX [4] standards and narrowband IoT (NB-IoT) [5], where the latter technology is recognised as a licensed resource in low power wide area (LPWA) communications. Unlike traditional multi-carrier systems, NBIoT tends to have fewer subcarriers and low-order modulation scheme such as binary phase shift keying (BPSK). A drawback of NB-IoT is that its limited bandwidth and transmission rate constrain its applications; unlike LTE-M [6], mission-critical applications are not suited to NB-IoT. This work is motivated by the current interest in extending NB-IoT applications by either increasing the information rate [7] or improving performance [8]. In this work, we focus on increasing the data rate, within NB-IoT limited bandwidth, so rates close to those of LTE-M can be obtained.

Over the past few years, there has been increased interest in non-orthogonal waveform techniques by academia and industry, aiming to approach the capacity limits [9]. In this area, a prominent contribution, termed as Faster-than-Nyquist (FTN) signalling, was firstly proposed by Mazo in 1975 [10], showing a $25 \%$ bandwidth efficiency gain without BER degradation. Spectrally Efficient FDM (SEFDM) is another notable technique that achieves bandwidth reduction. This technique was initially proposed by Rodrigues and Darwazeh in 2003 [11] and shows a greater than $25 \%$ bandwidth efficiency improvement [12]. Unfortunately, the self-introduced intercarrier interference (ICI) degrades the BER performance in this system but this can be tackled at the expense of transceiver complexity. A special case of SEFDM system, termed as Fast-OFDM, offers doubled bandwidth efficiency compared to OFDM without BER degradation [13], but only using singledimensional modulation. Fast-OFDM has been used in both wireless [14] and optical [15] communication systems.

Amongst existing techniques in wireless communications, Hilbert transform promises significant bandwidth efficiency enhancement due to its unique mathematical properties. This operation was initially used to define the analytic signal, which is a complex signal that specifies instantaneous amplitude and frequency [16]. In 1962, Bedrosian proposed the analytic signal representation for the single-sideband (SSB) modulation [17], which offers generalised formulation to obtain the onesided spectrum.

Due to its property of quadrature filtering, Hilbert transform is used in carrier-less amplitude and phase (CAP) modulation. In CAP modulation, finite impulse response (FIR) filters are employed to form a Hilbert pair [18]. The in-phase/quadrature (I/Q) components are separated, filtered and finally added up before transmission.

Inspired by the CAP modulation, in this work, we employ the orthogonal Hilbert transform pair and the Fast-OFDM scheme concurrently. Specifically, we propose to split the symbols to be transmitted into two parts; each is modulated and then filtered independently. The Hilbert filter pair is applied on two independent paths with time-reversed matched 
filters deployed at the receiver. Theoretically, twice the data rate is achieved by deploying Fast-OFDM signalling format followed by a further data rate doubling due to the Hilbert superposition. Simulation results show a quadrupled data rate compare to OFDM without error degradation.

The rest of the paper is organised as follows: section II details the principles of Fast-OFDM and Hilbert transform pair; section III addresses the generation of the proposed system model and describes the receiver side with the focus of the matched filtering; then in section IV, simulation results are discussed to show the system performance; finally, section $\mathrm{V}$ draws the conclusion.

\section{WAVEFORM DESIGN}

\section{A. Waveform Compression: Fast-OFDM}

Fast-OFDM is a variation of OFDM with a halved subcarrier separation. This leads to a significant advantage that, when compared to OFDM, the bandwidth efficiency of Fast-OFDM is doubled when the same modulation scheme is utilised. Single dimensional modulation scheme such as BPSK and MAmplitude Shift Keying (M-ASK) are applied in the scheme in order to achieve the same BER performance as OFDM. The continuous-time Fast-OFDM signal is given by [13]:

$$
x(t)=\frac{1}{\sqrt{T}} \sum_{k=-\infty}^{+\infty} \sum_{n=0}^{N-1} X_{n, k} e^{j 2 \pi n t / 2 T},
$$

where $T$ represents the period of an OFDM symbol, the subcarrier spacing of Fast-OFDM is equal to $1 / 2 T$ in (1), $N$ is the total number of the subcarriers, $n \in[0, N-1]$ denotes the frequency index of the subcarrier and $X_{n, k}$ represents the symbol transmitted on the $n^{\text {th }}$ subcarrier and the $k^{\text {th }}$ signalling interval.

Unlike higher level modulation scheme such as QPSK or 16-QAM, where I/Q components of the subcarriers are used concurrently, BPSK only adopts the I component to carry the data. As a result, Fast-OFDM system using BPSK violates the orthogonality among multiple subcarriers though, the transmitted signal does not suffer the introduced ICI as proved in the following.

The correlation of two arbitrary subcarriers indicates the ICI, given by:

$$
\begin{aligned}
R_{c}(m, n)= & \frac{1}{T} \int_{0}^{T} e^{j 2 \pi m t / 2 T} e^{j 2 \pi n t / 2 T} \\
= & \operatorname{sinc}(m-n) \\
& +j \sin \left(\frac{\pi(m-n)}{2}\right) \operatorname{sinc}\left(\frac{(m-n)}{2}\right),
\end{aligned}
$$

where $e^{j 2 \pi m t / 2 T}$ and $e^{j 2 \pi n t / 2 T}$ are the $m^{t h}$ and the $n^{t h}$ subcarrier respectively, $1 / 2 T$ represents the subcarrier separation as stated in (1). Extracting the real and imaginary part of the correlation, we have:

$$
\Re\left\{R_{c}\right\}=\operatorname{sinc}(m-n)
$$

for the real part of the correlation and
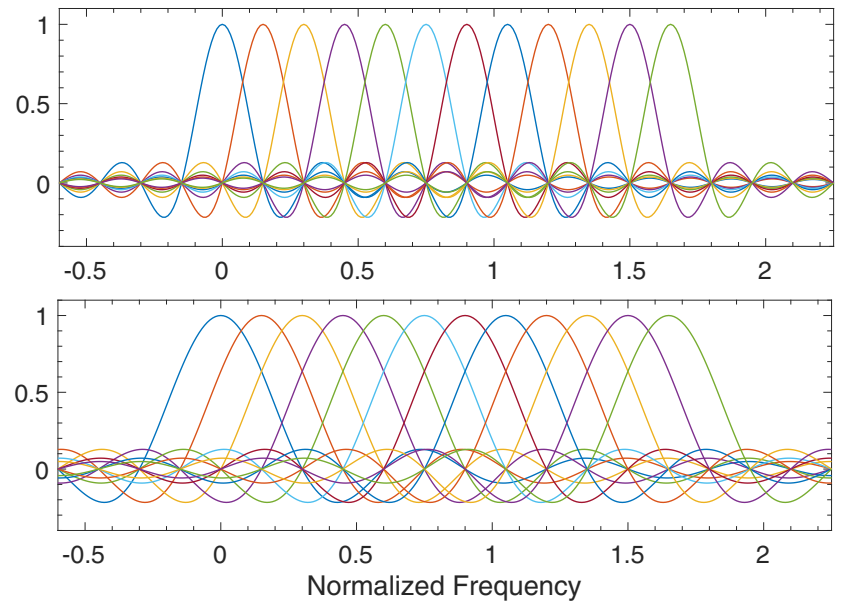

Fig. 1. OFDM and Fast-OFDM subcarriers representations $(N=12)$ with identical spectrum occupancy while Fast-OFDM provides twice the data rate of OFDM

$$
\Im\left\{R_{c}\right\}=\sin \left(\frac{\pi(m-n)}{2}\right) \operatorname{sinc}\left(\frac{(m-n)}{2}\right)
$$

for the imaginary part, where $\Re$ and $\Im$ denote the real and imaginary part of the complex expression, respectively, and the sinc function is defined as $\operatorname{sinc}(x)=\sin (\pi x) / x$. It is clear from the equations that, for the real part, the self-correlation (i.e. when $m=n$ ) is zero and the cross-correlation (i.e. when $m \neq n)$ is one. Even though the cross-correlation for the imaginary part is not equal to zero, the orthogonality rule is still valid since no data are carried on this dimension. Therefore, by using such signal format, the ICI can be removed for the received signal.

Figure 1 compares the spectrum of both OFDM and FastOFDM signal where parallel sinc pulses are used as representations of 12 subcarriers for both systems. It can be seen that the underlying occupied bandwidth of Fast-OFDM signal is same as that of OFDM. However, Fast-OFDM provides a doubled data rate with the identical BER performance of ideal OFDM achieved under the same modulation.

\section{B. Filtering: Hilbert Transform pair}

The analytic signal $g_{+}(t)$ is a complex signal that can be expressed as [16]:

$$
g_{+}(t)=g(t)+j * \hat{g}(t) .
$$

The I component $g(t)$ and Q component $\hat{g}(t)$ of the analytic signal, termed as an Hilbert transform pair, are signals in a relation that $\hat{g}(t)$ is the Hilbert transform of $g(t)$. This can be expressed as [16]:

$$
\hat{g}(t)=\mathcal{H}\{g(t)\},
$$

where $\mathcal{H}[\cdot]$ denotes the Hilbert transform operation. The significant feature of the Hilbert pair is that $g(t)$ and $\hat{g}(t)$ are orthogonal, thus giving the equation below [16]:

$$
\langle g(t), \hat{g}(t)\rangle=0,
$$


where $\langle\cdot, \cdot\rangle$ denotes an inner product. Based on the orthogonality, we apply the Hilbert pair as pulse shaping filters in this work to double the data rate under reliable transmissions.

The orthogonal filter pair can be achieved by the product of a Nyquist or root-Nyquist filtering pulse and an orthogonal Hilbert pair. In this work, we employ the orthonormal sinusoidal signal carrier pair as the basis.

\section{SySTEM MODELLING}

In order to meet the NB-IoT scenario, we use 12 subcarriers in the proposed system with the overall bandwidth of $180 \mathrm{kHz}$ [5]. The system modelling focuses on the filtering structure design where Hilbert pair and its matched filter pair are used on both transmission and reception sides. Numerical expressions are given to clarify the processing procedures. The Additive White Gaussian Noise (AWGN) channel is considered in this case.

\section{A. Transmitter Scheme}

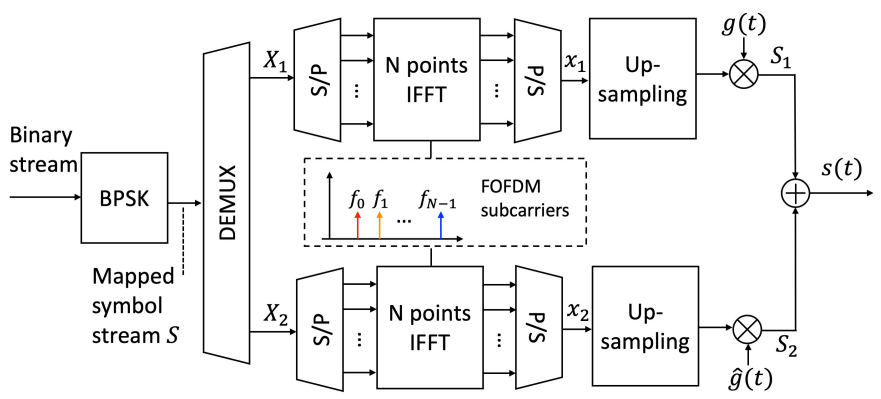

Fig. 2. Simplified block diagram of HT-Fast-OFDM transmitter; subcarriers occupy the same frequency range on two separate paths

Figure 2 illustrates the transmitter block diagram of the proposed Fast-OFDM system using Hilbert pulse pair. The pseudo-randomly generated bit stream is input into the BPSK mapper block, where the binary bits are transferred to bipolar symbols. The demultiplexer split the mapped symbol stream $S$ equally, therefore yielding two sub-streams $X_{1}$ and $X_{2}$. On two independent paths, the symbols are processed through identical blocks. For each path, the serial symbols are paralleled. Then the $N$ points IFFT is used to modulate these symbols to the 12 subcarriers. Once the Fast-OFDM signals are generated, the next block performs up-sampling by inserting $q-1$ zeros between the adjacent symbols, wherein $q=4$ is the up-sampling factor. The up-sampled symbols are then filtered by the Hilbert filter pair, giving $S_{1}$ and $S_{2}$. The summation of the filtered signals on two paths output the transmitted signal $s(t)$, given by:

$$
s(t)=S_{1}(t)+S_{2}(t) .
$$

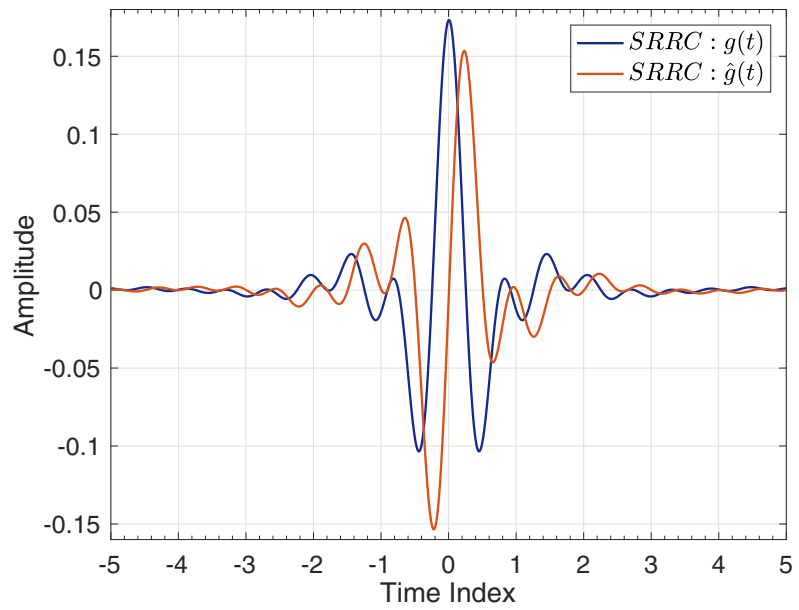

Fig. 3. Hilbert SRRC pulse pair in time domain $(\mathrm{Ls}=10, \beta=0.35)$

The Hilbert transform pair, namely the $g(t)$ and $\hat{g}(t)$, are generated by a square root raised cosine (SRRC) pulse $p(t)$ in the proposed system, which is expressed as [19]:

$$
p(t)=\frac{2 \beta\left[\cos \left(\frac{(1+\beta) \pi t}{T_{s}}\right)+\sin \left(\frac{(1-\beta) \pi t}{T_{s}}\right)\left(\frac{4 \beta t}{T_{s}}\right)^{-1}\right]}{\pi \sqrt{T_{s}}\left[1-\left(\frac{4 \beta t}{T_{s}}\right)^{2}\right]} .
$$

The digital filter pair applied on two separate paths with the carrier frequency of $f_{c}$, is given by:

$$
g(t)=p(t) \cos \left(2 \pi f_{c} t\right), \quad \hat{g}(t)=p(t) \sin \left(2 \pi f_{c} t\right),
$$

where Ts is the symbol period, $\beta \in[0,1]$ is the roll-off factor of the SRRC pulse that controls the excess bandwidth of the pulse spectrum. The filter bandwidth can be achieved by $B=$ $(1+\beta) / 2 T_{s}$. Figure 3 shows the time domain representation of the HT filter pair used in this work, with the filter length of $L_{s}=10$ and the roll-off factor $\beta=0.35$, which are chosen based on the investigations in [19].

\section{B. Receiver Scheme}

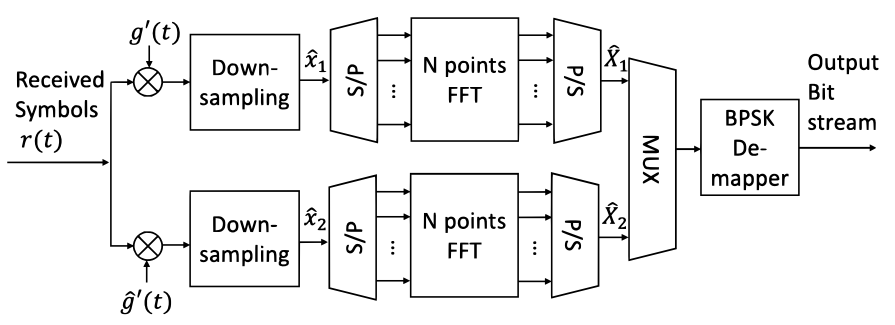

Fig. 4. Simplified block diagram of HT-Fast-OFDM receiver

Figure 4 shows the receiver's architecture of the proposed system. Matched filters (MF) are employed to demodulate the received signals, followed by a hard decision detector. The matched filtering procedure consists of two stages: separate the received symbol to two streams that correspond to the output 
symbols on the independent transmitter paths; demodulate each symbol streams using FFT operation. The first stage involves the correlation of the received signal with the matched filter pair, which is the time-reversed form of the Hilbert pulse pair employed at the transmitter. The matched filter pair can be expressed as [19]:

$$
g^{\prime}(t)=g(-t), \quad \hat{g}^{\prime}(t)=\hat{g}(-t) .
$$

Note that the matched filter pair are orthogonal as it is stated in (7) and hence enabling the recovery of the Hilbert superposition at the transmission side.

Moving to the second stage, the separated signals are then down-sampled and input into the FFT block, where demodulation take place in a more efficient way for implementation. After the hard decision, bipolar symbol streams on both paths are achieved and subsequently the serial symbols are demapped to give the recovered bit stream.

\section{Performance Investigations}

System performance are investigated in terms of the spectrum property, BER performance and effective data rate. Apart from the aforementioned system model, simulations in MATLAB are carried out also adopting the typical Fast-OFDM system as well as OFDM systems that use both BPSK and 16QAM modulation schemes for comparison.

\section{A. Spectrum Illustration}

For clear demonstration and comparison, the spectrum is plotted with the power spectral density normalised in $\mathrm{dB}$. The $E_{b} / N_{0}$ in simulation is selected to be $10 \mathrm{~dB}$ as the corresponding BER reaches beyond $10^{-4}$. Fig. 5 compares the spectrum of the OFDM BPSK signal and the proposed HT-Fast-OFDM signal. It can be seen that two spectra occupy the same bandwidth (i.e. $180 \mathrm{kHz}$ ). As expected, the proposed transmitted signal is a superposition of two independent signal streams; each obtains the data rate of $360 \mathrm{kbits} / \mathrm{s}$. Therefore, the transmitted data rate is equal to $720 \mathrm{kbits} / \mathrm{s}$, which is 4 times that of the OFDM BPSK signal. The achievable data rate is also discussed in next sections in relation to the BER performance. Furthermore, the spectral efficiency $\eta$ intuitively provides a measurement of the bit rate improvement of the proposed signal, termed as the ratio of the data rate and the occupied bandwidth.

\section{B. Bit Error Rate}

Theoretically, based on the definition in section II, FastOFDM can achieve higher data rate without error penalties when the transmitted signal occupies the same bandwidth as OFDM. The Hilbert pulse pair, attributed to its orthogonality feature, allows the superposition of two independent symbols to be separated without interference added.

The results presented in Fig. 6 show the BER performance of different systems with respect to the varying value of $E_{b} / N_{0}$ regimes. Wherein, the BER curve of the proposed system is generated by employing the transceiver architecture in Fig. 2 and Fig. 4. A significant observation from the figure is that

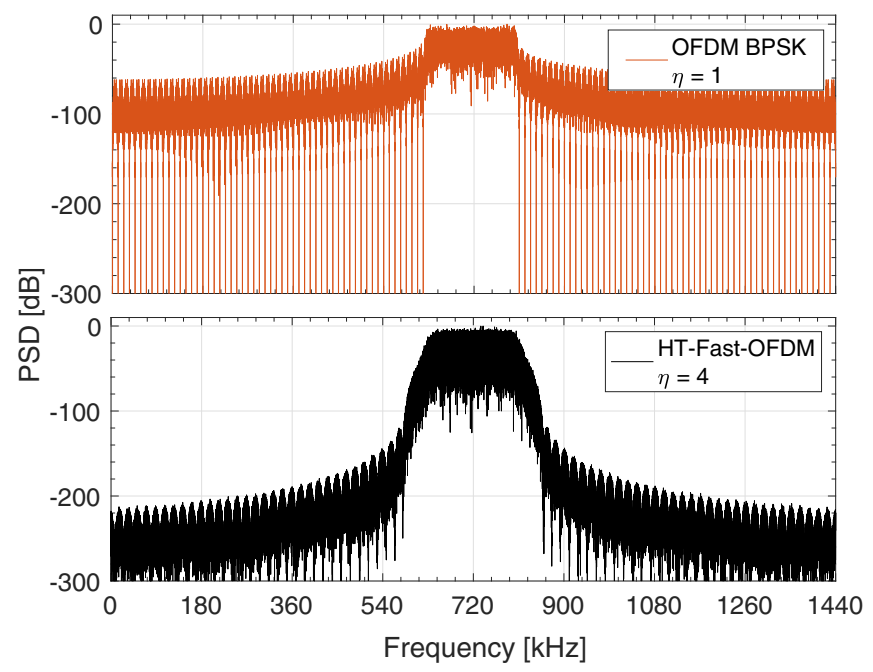

Fig. 5. Spectrum comparison: OFDM signal for BPSK modulation (upper); Fast OFDM signal using Hilbert SRRC pair $(N=12, \beta=0.35)$

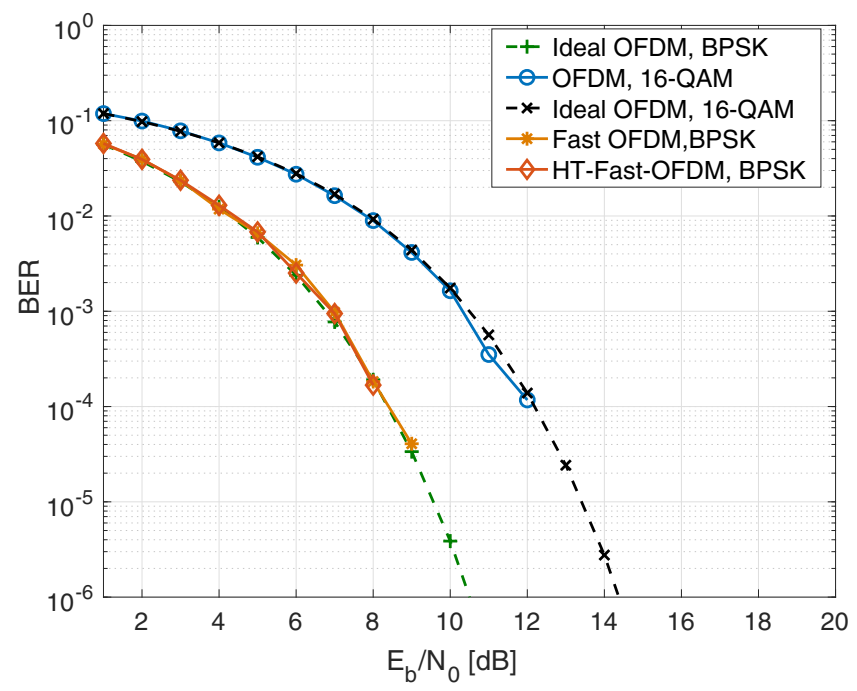

Fig. 6. BER performance for HT-Fast-OFDM (BPSK) in comparison with other OFDM and Fast-OFDM systems

the Fast-OFDM signal using the Hilbert filter pair scheme has the same BER performance as the typical Fast-OFDM signal as well as the BPSK OFDM signal. Therefore, a compelling advantage of the Fast-OFDM system using Hilbert filter pair can be seen that better BER performance is achieved when transmitting the same data rate.

\section{Data Rate Comparison}

The effective data rate is defined as the achievable bit rate in condition of a fixed bandwidth consumption with a certain value of BER reserved. The numerical expression of effective data rate is given by [14]:

$$
R_{e}=(1-B E R) \times R_{s} \times \log _{2} O \times\left(N_{d} / N\right)
$$


where BER represents the metric bit error rate. This value is used to evaluate the data rate of the aforementioned systems. Additionally, in the equation, $R_{s}$ is the symbol rate, $O$ denotes the constellation cardinality, $N_{d}$ is the number of data subcarriers and $N$ is the total number of subcarriers. Since no cyclic prefix nor guard band is utilised in the proposed system, the ratio of $N_{d}$ and $N$ equals one. Table I compares the effective data rate of the proposed system with other three systems. It is assumed that in the simulations all the other specifications for various systems are identical.

TABLE I

MAXIMUM DATA RATE

\begin{tabular}{c|c|c|c}
\hline \hline System & $\begin{array}{c}\text { Modulation } \\
\text { Scheme }\end{array}$ & $\begin{array}{c}\text { Effective } \\
\text { Data Rate } \\
{[\mathrm{kbits} / \mathrm{s}]}\end{array}$ & $\begin{array}{c}\text { Spectral } \\
\text { Efficiency } \\
{[\mathrm{bits} / \mathrm{s} / \mathrm{Hz}]}\end{array}$ \\
\hline OFDM & BPSK & 180 & 1 \\
Fast-OFDM & BPSK & 360 & 2 \\
OFDM & 16-QAM & 720 & 4 \\
HT-Fast-OFDM & BPSK & 720 & 4 \\
\hline
\end{tabular}

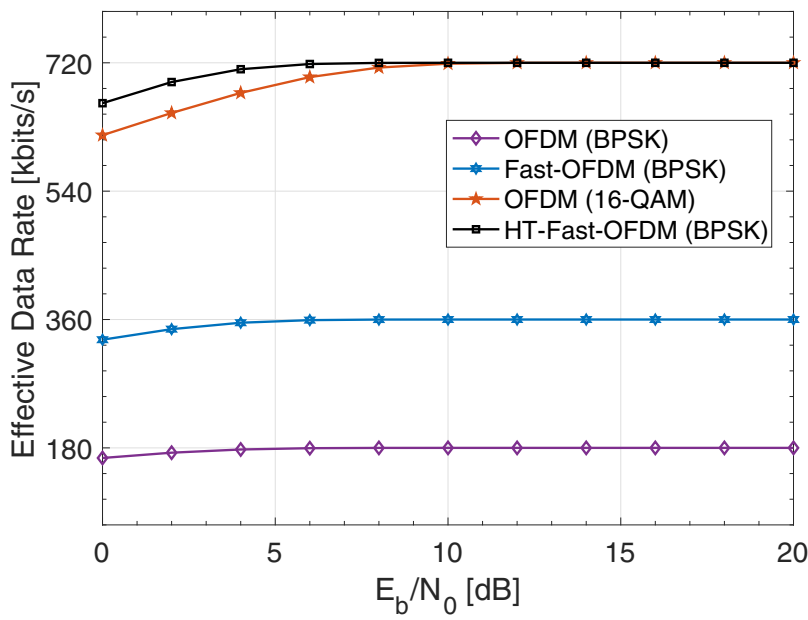

Fig. 7. Effective Data Rate Comparison: typical OFDM system for 16-QAM and BPSK modulation, Fast-OFDM BPSK system and the proposed system.

Figure 7 depicts the measured effective data rate of the systems in relation to the varying value of $E_{b} / N_{0}$. As expected, the proposed system transmits four times the data rate of the BPSK OFDM system as well as twice that of the Fast-OFDM system. Since the error penalty is considered, the 16-QAM OFDM system theoretically offers the same data rate as the proposed system though, achieves lower effective data rate as it approaches but cannot reach $720 \mathrm{kbits} / \mathrm{s}$ as the value of $E_{b} / N_{0}$ increases.

\section{Conclusions}

In this paper, a new signalling method is proposed in NBIoT scenario so that quadrupled data rate can be achieved compared to conventional OFDM. In the designed system, we employ Fast-OFDM scheme and Hilbert transform pair to increase the data rate under reliable transmission within a limited bandwidth of $180 \mathrm{kHz}$. Numerical analysis of the transceiver structure shows the orthogonality feature of both techniques, which enables the recovery of received signals without error penalties. Investigations are carried out to compare the proposed system with both Fast-OFDM system and OFDM with BPSK and 16-QAM schemes. Results show that the proposed system outperforms other systems, offering four times the data rate of OFDM with the same modulation scheme.

\section{REFERENCES}

[1] F.-L. Luo and C. Zhang, Signal processing for 5G: algorithms and implementations. John Wiley \& Sons, 2016.

[2] E. Dahlman, H. Ekstrom, A. Furuskar, Y. Jading, J. Karlsson, M. Lundevall, and S. Parkvall, "The 3g long-term evolution-radio interface concepts and performance evaluation," in Vehicular Technology Conference, 2006. VTC 2006-Spring. IEEE 63rd, vol. 1. IEEE, 2006, pp. 137-141.

[3] E. Perahia, "Ieee $802.11 \mathrm{n}$ development: History, process, and technology," IEEE Communications Magazine, vol. 46, no. 7, 2008.

[4] W. Forum, "Mobile WiMAX-A Technical Overview and Performance Evaluation," 2006. [Online]. Available: http://wimaxforum.org/news/ downloads/Mobile_WiMAX_Part1_Overview_and_Performance.pdf

[5] M. Chen, Y. Miao, Y. Hao, and K. Hwang, "Narrow band internet of things," IEEE Access, vol. 5, pp. 20 557-20 577, 2017.

[6] "LTE; Evolved Universal Terrestrial Radio Access (E-UTRA)," Physical Layer Procedures, vol. Rel. 14, no. 3GPP Standard TS 36.213 v.14.2.0, Apr 2017.

[7] T. Xu, C. Masouros, and I. Darwazeh, "Waveform and space precoding for next generation downlink narrowband iot," IEEE Internet of Things Journal, 2019.

[8] Y. Huang, B. Su, and I.-K. Fu, "Heterogeneous 1te downlink spectrum access using embedded-gfdm," in 2016 IEEE International Conference on Communications Workshops (ICC). IEEE, 2016, pp. 474-479.

[9] D. Rainnie, Y. Feng, and J. Bajcsy, "On capacity merits of spectrally efficient fdm," in MILCOM 2015-2015 IEEE Military Communications Conference. IEEE, 2015, pp. 581-586.

[10] J. E. Mazo, "Faster-than-nyquist signaling," Bell System Technical Journal, vol. 54, no. 8, pp. 1451-1462, 1975.

[11] M. Rodrigues and I. Darwazeh, "A spectrally efficient frequency division multiplexing based communications system," in Proc. 8th Int. OFDM Workshop, 2003, pp. 48-49.

[12] I. Darwazeh, H. Ghannam, and T. Xu, "The first 15 years of sefdm: A brief survey," in 2018 11th International Symposium on Communication Systems, Networks \& Digital Signal Processing (CSNDSP). IEEE, 2018, pp. 1-7.

[13] M. Rodrigues and I. Darwazeh, "Fast OFDM: A proposal for doubling the data rate of OFDM schemes," 2002.

[14] T. Xu and I. Darwazeh, "Non-orthogonal waveform scheduling for next generation narrowband iot." IEEE, 2018.

[15] P. A. Haigh and I. Darwazeh, "Visible light communications: Fastorthogonal frequency division multiplexing in highly bandlimited conditions," in 2017 IEEE/CIC International Conference on Communications in China (ICCC Workshops). IEEE, 2017, pp. 1-8.

[16] M. M. Alavi-Sereshki, "Analytic signals and hilbert transforms," Ph.D. dissertation, Texas Tech University, 1972.

[17] E. Bedrosian, "The analytic signal representation of modulated waveforms," Proceedings of the IRE, vol. 50, no. 10, pp. 2071-2076, 1962.

[18] P. A. Haigh, S. T. Le, S. Zvanovec, Z. Ghassemlooy, P. Luo, T. Xu, P. Chvojka, T. Kanesan, E. Giacoumidis, P. Canyelles-Pericas et al., "Multi-band carrier-less amplitude and phase modulation for bandlimited visible light communications systems," IEEE Wireless Communications, vol. 22, no. 2, pp. 46-53, 2015.

[19] P. A. Haigh, P. Chvojka, S. Zvánovec, Z. Ghassemlooy, and I. Darwazeh, "Analysis of Nyquist Pulse Shapes for Carrierless Amplitude and Phase Modulation in Visible Light Communications," Journal of Lightwave Technology, vol. 36, no. 20, pp. 5023-5029, 2018. 